\title{
Pérou: Les gestionnaires doivent régulièrement veiller á la qualité des soins
}

Frontiers in Reproductive Health

Follow this and additional works at: https://knowledgecommons.popcouncil.org/departments_sbsr-rh

Part of the International Public Health Commons, Quality Improvement Commons, and the Women's Health Commons How does access to this work benefit you? Let us know!

\section{Recommended Citation}

"Pérou: Les gestionnaires doivent régulièrement veiller á la qualité des soins," FRONTIERES Résumés de Recherche Opérationnelle. Dakar: Population Council, 2000. 


\section{Pérou}

Qualité des soins

$N^{\circ} 6$

\section{Les gestionnaires doivent régulièrement veiller à la qualité des soins}

En référence aux directives nationales sur les soins, plus de 90 pourcent des agents traitent leurs patientes avec respect et leur proposent un large éventail d'options contraceptives. Néanmoins, ils auraient dû mieux faire, dans l'amélioration de la qualité des soins de leurs clientes en leur donnant davantage d'informations sur l'utilisation adéquate de la méthode choisie et ses éventuels effets secondaires. Une norme de qualité à 100 pourcent devrait être établie pour respecter les droits individuels des clientes à la reproduction.

\section{Contexte}

A la fin des années 1980, le Ministère de la Santé (MS) a assigné des objectifs spécifiques, selon la méthode, à des groupes de structures de santé. En 1998, le MS a changé de politique afin de s'assurer, qu'en matière de santé de la reproduction, les services correspondaient aux besoins et attentes des individus. Il a mis un terme aux quotas des méthodes, décidé de l'arrêt des campagnes sur la Contraception Chirurgicale Volontaire $(\mathrm{CCV})$, et élaboré des normes destinées à garantir la qualité des soins et un choix éclairé.

En 1999, le Population Council a collaboré avec le MS pour déterminer si les prestataires se conformaient aux nouvelles directives, et aussi pour élaborer un système de suivi qui permette de vérifier cette conformité au fil du temps.

\section{Résultats}

- Plus de 90 pourcent des prestataires en planification familiale (PF) du MS traitent leurs clientes avec respect et offrent une variété d'options contraceptives sans favoriser une méthode particulière.
- Cependant, les prestataires du MS peuvent faire plus pour s'assurer que chaque client(e) reçoit une information adéquate sur la méthode qu'il/elle a choisie. Par exemple la plupart des prestataires ont prévenu les clientes simulées des changements possibles de leur cycle menstruel associés aux injectables DEPO-PROVERA, mais ils n'ont pas mentionné l'éventualité d'un retour tardif de la fécondité après l'arrêt de la méthode. Les clientes réelles ont montré, après leurs entretiens, qu'elles avaient une bonne information sur la pilule, le préservatif, les injectables et la CCV. Cependant les connaissances spécifiques relatives à la méthode choisie ou utilisée étaient incomplètes.

- La plupart des prestataires des centres urbains n'ont pas vérifié auprès des clientes simulées, l'existence de trois des quatre conditions médicales pour lesquelles l'utilisation de DEPO-PROVERA était contre indiquée. Peu de prestataires des centres de santé ont fourni des informations sur les symptômes qui requièrent une attention médicale.

- Plus de 90 pourcent des clientes stérilisées dans les hôpitaux ont déclaré avoir pris ellesmêmes la décision ou conjointement avec le prestataire, et 98 pourcent connaissaient ses conséquences en matière de reproduction. 
- Dans les centres de santé urbains, les clientes simulées ont bénéficié de counseling pendant 2 à 45 minutes. Les informations transmises par les prestataires pendant les séances de 9 à 14 minutes dépassent de 43 pourcent celles transmises durant les séances de 2 à 8 minutes.

- Lors des entretiens à domicile, la plupart des clientes des postes de santé ont déclaré qu'elles avaient elles-mêmes décidé d'utiliser la contraception, et plusieurs d'entre elles avaient choisi une méthode spécifique avant de se rendre au centre de santé. Les clientes des zones rurales avaient cependant une connaissance limitée de la méthode choisie.

\section{Implications pour les programmes}

- Le MS a élaboré et fait diffuser de nouvelles normes de qualité des soins et renforcé la stratégie de recyclage et de supervision de ses prestataires.
- Les structures du MS et les prestataires individuels doivent être évalués sur la qualité de leur performance et doivent bénéficier d'un retour d'information de manière régulière.

- Les prestataires doivent consacrer tout le temps nécessaire aux échanges avec les clientes.

- Une norme de qualité à 100 pourcent doit être la règle, afin de respecter les droits individuels des clientes à la reproduction.

- Deux des méthodes de collecte de donnéesl'entretien à la sortie de la cliente et l'utilisation de clientes simulées qui demandent un DEPOPROVERA se sont révélées fiables pour le contrôle de qualité des soins dans les centres de santé. Les outils de contrôle à la disposition des hôpitaux et centres de santé des zones rurales doivent encore être améliorés.

\section{Procédé de recherche}

Afin de gagner du temps et d'économiser de l'argent, l'étude a utilisé un Echantillonnage par Tirage Aléatoire (ETA) pour constituer un échantillon de 6589 points de prestations de services du Pérou. L'échantillon a consisté en 19 hôpitaux, 19 centres de santé, et 19 postes de santé. Six observations ont été effectuées dans chaque structure. Les données ont été collectées de juin à août 1999 de la manière suivante :

- Les hôpitaux ont été évalués sur la base de rapports des clientes simulées qui ont sollicité un counseling en CCV et sur la base des entretiens à domicile avec les utilisatrices de CCV.

- Les centres de santé ont été évalués sur la base de rapports des clientes fictives qui ont sollicité des injectables DEPO-PROVERA et des interviews de sortie de consultation des clientes de planification familiale (PF).

- Les postes de santé en zones rurales ont été évalués sur la base d'entretiens à domicile, avec de nouvelles clientes PF.

Pour qu'un élément satisfasse aux normes ETA, au moins 95 pourcent des six clientes interrogées dans chaque structure devraient bénéficier d'informations et de services spécifiques de la part du prestataire dans au moins 80 pourcent des structures de l'échantillon. Des analyses parallèles ont été effectuées sur la base de 95 pourcent d'intervalle de confiance pour chaque échantillon $(\mathrm{N}=114)$.

Leon, Federico R.,1999. «Peru : Respect des normes et de la qualité des soins par les prestataires ».

Leon, Federico R., et al, 1999. «Durée des séances de counseling et volume d'informations échangées dans les hôpitaux péruviens ». Pour de plus amples informations contacter : Population Council, Av. San Borja Sur 676, Lima 43, Peru. Tel. 511475-0275; Fax: 511-475-0675; E-mail: pclima@amauta.rcp.net.pe or contact: Population Council, Escondida 110, Villa Coyoacán, 04000, Mexico, D.F. Mexico. Tel. 52-5659-8537; Fax: 52-5554-1226; E-mail: disemina@ popcouncil.org.mx. Ce projet a été réalisé avec le concours de l'AGENCE AMERICAINE POUR LE DEVELOPPEMENT INTERNATIONAL (USAID) aux termes du contrat de coopération HRN-A-00-98-00012-00. 